\title{
ANÁLISE DO MERCADO DE TRABALHO ODONTOLÓGICO NA REGIÃO NORDESTE DO BRASIL
}

\author{
AN ANALYSIS OF THE DENTISTRY JOB MARKET IN THE NORTHEAST REGION OF BRAZIL
}

\author{
Luiz Renato Paranhos* \\ Ivan Delgado Ricci ** \\ Danilo Furquim Siqueira *** \\ Marco Antonio Scanavini **** \\ Eduardo Daruge Júnior ${ }^{* * * * * *}$
}

\begin{abstract}
RESUMO
Introdução: O objetivo deste trabalho é avaliar o mercado de trabalho do cirurgião-dentista, nas diferentes especialidades, na Região Nordeste do Brasil, além de avaliar a relação cirurgião-dentista/habitante e especialista/habitante, visando melhorar a compreensão dos rumos da profissão, direcionando a atuação profissional. Material e Métodos: Foram coletados dados do Conselho Federal de Odontologia e do Instituto Brasileiro de Geografia e Estatística. Resultados: Os Estados da Regiáo Nordeste do Brasil apresentam índices maiores que o recomendado pela Organização Mundial de Saúde (1:1.500), com exceçáo da Paraíba e do Rio Grande do Norte que apresentam maior proporçấo entre habitantes por CD. A Região Nordeste concentra 13,59\% do total de cirurgióes-dentistas de todo o Brasil. Conclusóes: O mercado de trabalho para os especialistas da Região Nordeste nas áreas de Prótese Buco-Maxilo-Facial, Odontologia do Trabalho, Odontologia Legal, Patologia Bucal e Estomatologia apresenta-se com melhor proporção de especialista/ habitante, enquanto as áreas de Ortodontia, Endodontia e Prótese Dentária apresentam maior proporção de especialista/habitante.
\end{abstract}

DESCRITORES: Mercado de trabalho • Exercício profissional • Assistência odontológica • Educação em Odontologia.

\begin{abstract}
Introduction: This work aims to evaluate the job market for dental surgeons, within their different specialties, in the Northeast region of Brazil, in addition to evaluating dental surgeon/inhabitant and specialist/ inhabitant ratios. This study aims to better understand the trends in this profession, and also suggests fields of professional activity. Materials and Methods: Data was collected from the Brazilian National Council of Odontology and the Brazilian National Institute of Geography and Statistics. Results: The states within the Northeast region of Brazil have higher professional/inhabitant ratio levels than recommended by the World Health Organization (1:1,500), with the exceptions of the states of Paraíba and Rio Grande do Norte, which feature higher inhabitant/surgeon ratios. This region concentrates $13.59 \%$ of all dental surgeons in Brazil. Conclusions: The job market for specialists in the Northeast region in the fields of Bucomaxillofacial Prosthetics, Workplace Dentistry, Forensic Dentistry, Mouth Pathology and Stomatology features the best specialist/inhabitant ratio, while the fields of Orthodontics, Endodontics and Dental Prosthetics have higher specialist/inhabitant ratios.
\end{abstract}

DESCRIPTORS: Job market $\bullet$ Professional practice $\bullet$ Dental care $\bullet$ Education, dental

\footnotetext{
* Especialista em Ortodontia - AMO/Dental Press. Mestre e Especialista em Odontologia Legal e Deontologia - Faculdade de Odontologia de Piracicaba (FOP/UNICAMP)

** Especialista em Saúde Coletiva - CPO São Leopoldo Mandic. Aluno do Programa de Pós-Graduação em Odontologia da Universidade Metodista de São Paulo (UMESP) - Mestrado em Ortodontia

*** Mestre e Doutor em Ortodontia. Professor Titular do Programa de Pós-Graduação em Odontologia da Universidade Metodista de São Paulo (UMESP)

**** Mestre e Doutor em Ortodontia. Coordenador do Programa de Pós-Graduaçáo em Odontologia da Universidade Metodista de São Paulo (UMESP). Diretor da Faculdade de Odontologia da UMESP

***** Professor Doutor de Odontologia Legal da Faculdade de Odontologia de Piracicaba (FOP/UNICAMP)
} 
Paranhos LR, Ricci ID, Siqueira DF, Scanavini MA, Daruge Júnior E. Análise do mercado de trabalho odontológico na região nordeste do Brasil. Revista de Odontologia da Universidade Cidade de São Paulo 2009 mai-ago; 21(2): 104-18

\section{INTRODUÇÃO E REVISÃO DA LITERATURA}

Em meados do século XIX, a Odontologia foi separada da medicina, transformando-se em uma profissão independente. Até então, essa área da saúde era realizada por pessoas que adquiriam conhecimentos exclusivos de forma prática, inicialmente, quando do predomínio de açôes voltadas para a cosmética, sendo apenas realizadas extraçóes e reposição dos dentes. Posteriormente, a forma de atuação tornou-se teórico-prática, quando da mudança da etapa empírica para a científica, momento em que as açóes tiveram uma mudança de foco, entrando na atuação terapêutica. Nesse estágio, os praticantes da arte dentária deixam de ser meros "tiradentes" ou fabricantes de "dentes artificiais", abandonando o treinamento empírico e o trabalho mecânico.

O momento foi importante, também, pois deu-se início à organização profissional, iniciando uma disputa pelo mercado crescente. Foi o início da ciência nesta profissão, vinculada à sua escolarização, legitimada pela inauguração da primeira escola de Odontologia em Baltimore, nos Estados Unidos, em 1839.

Com o passar do tempo até os dias atuais, ocorreu uma mercantilização da profissão, fato este que resultou no processo de esgotamento do modelo tradicional de educação superior nas áreas de saúde. Aliada a isso, uma somatória de problemas resultou na diminuiçáo do interesse pelos cursos de Odontologia, problemas estes relacionados à perda de prestígio social do cirurgião-dentista bem como baixa remuneração da profissão, escassez de empregos, taxas de regulamentação da profissão, impostos e mercado profissional, que, a cada dia, apresenta-se mais competitivo.

Houve decréscimo anual do número de cirurgiōesdentistas recém-formados, pois, há sete anos, 12.000 formandos lançavam-se no mercado de trabalho anualmente (Carvalho e Orlandi ${ }^{2}$ 2001, Farias ${ }^{7}$ 2006, Sória et al. ${ }^{23}$ 2002) e hoje, o número aproxima-se de 9.000 profissionais. Somados aos 220.136 cirurgióes-dentistas (CDs) já existentes, e devido à distribuição irregular por todo o território brasileiro, aliados à concorrência desleal e antiética do profissional, o angariamento e fidelização dos pacientes nos consultórios odontológicos estão cada vez mais difíceis, sendo necessário que o profissional adquira características empreendedoras (Sória et al. ${ }^{23}$ 2002).

A educação continuada é, de fato, indispensável para o profissional, pois proporciona uma oportunidade da reciclagem contínua, aprimorando os conhecimentos técnicos, científicos e práticos. Porém, a especialização acaba por fragmentar o conhecimento, tornando-se necessária a interdisciplinaridade profissional.

Dessa forma, o perfil profissional tem passado por constantes modificaçóes e é estudado com o objetivo de colaborar na atuação do profissional no mercado. Dentro dessa linha, Machado et al. ${ }^{15}$ (1992) ressaltaram que a maioria dos cirurgiôes-dentistas são autônomos, porém sofrendo um decréscimo na categoria, passando de 69,9\% em 1970, para 54,5\% em 1980. Concordando com esse estudo, Silva Filho e Eleutério ${ }^{22}$ (1977), realizaram um trabalho em Araraquara (SP), com profissionais formados no período de 1964 a 1974 e demonstraram que $61,1 \%$ atuam apenas em consultório particular.

O mercado de trabalho para o cirurgião-dentista começa a sofrer modificaçóes mais acentuadas a partir de 1980. Pereira e Botelho ${ }^{17}$, em 1997, realizaram uma pesquisa com 3.191 profissionais, constatando que $51 \%$ atuam como profissionais liberais, e $48 \%$ destes atendem por sistema de convênios, $45 \%$ trabalham no consultório e são assalariados e $49 \%$ são somente assalariados.

Galassi et al. ${ }^{8}$, em 2004, avaliaram o perfil atual do profissional, quando elaboraram um questionário direcionado a 900 cirurgióes-dentistas clínicos gerais, na Região Sudeste. Concluíram que há um grande interesse dos profissionais pelo aperfeiçoamento, mediante participação em eventos científicos. Consideraram que a competência e honestidade são fatores decisivos para a conquista e manutençấo dos clientes.

Ainda no mesmo ano, Koide et al. ${ }^{13}$ (2004) estudaram o perfil profissional de uma amostragem de CDs do corpo docente de uma universidade particular de São Paulo. A avaliação foi realizada por questionários semiestruturados distribuídos a 120 profissionais entre docentes e estagiários. Os autores concluíram que o CD atende, na grande maioria, em clínica particular, e que os convênios odontológicos não fazem parte da maior fonte de renda dos profissionais credenciados a essas instituições.

Gushi et al. ${ }^{10}$ (2004) avaliaram o perfil profissional de CDs formados no período entre 1960-1997, por meio de questionários emitidos a ex-alunos da Faculdade de Odontologia de Piracicaba - UNICAMP. Concluíram que o perfil se alterou ao longo dos tempos e que a alta competitividade e o alto preço de equipamentos aliados a pouca visão administrativa resultaram em desistências da própria carreira.

Em um trabalho realizado em 1999, Puricelli et al. ${ }^{19}$ 
Paranhos LR, Ricci ID, Siqueira DF, Scanavini MA, Daruge Júnior E. Análise do mercado de trabalho odontológico na região nordeste do Brasil. Revista de Odontologia da Universidade Cidade de São Paulo 2009 mai-ago; 21(2): 104-18

relataram existir um aumento da participação feminina no mercado de trabalho odontológico, parecendo seguir a tendência demográfica, que apontava uma pequena diferença entre a população masculina (77.447.541) e a feminina (79.632.032). Afirmaram, ainda, que a médio e longo prazo o sexo feminino poderá responder pela maior parte da Odontologia no Brasil.

Já preocupados sobre como a distribuição geográfica pode afetar a situaçáo do mercado de trabalho dos cirurgióes-dentistas, Rocha et al. ${ }^{20}$ (1985) fizeram um levantamento dos CDs em todos os Estados da Região Nordeste do Brasil. Concluíram que o mercado nas capitais nordestinas está excessivo, com um CD para 920 habitantes, em razão de fatores econômicos. Afirmaram haver 728 municípios nessa regiáo que não possuíam um único profissional, deixando assim um campo aberto para a prática ilegal da profissão.

Garcia et al. ${ }^{9}$ (1997) analisaram o mercado de trabalho das principais cidades do Estado de Santa Catarina, avaliando a proporção habitante/CD, objetivando mostrar uma opção para a localização do local de trabalho. De forma semelhante, Lara e Pereira Filho ${ }^{14}$, em 1998, investigaram a distribuição geográfica de 1050 cirurgióes-dentistas de Porto Alegre, resultando em subsídios aos profissionais quanto à escolha do local para a montagem de seus consultórios. Concluíram ainda que existe má distribuição dos consultórios dentro do município, pois o $\mathrm{CD}$, normalmente, busca a área central da cidade, por apresentar maior índice de desenvolvimento econômico.

Com o objetivo de analisar as relaçóes $\mathrm{CD} /$ habitante e especialista/habitante nos municípios do Estado do Paraná, e a distribuição das entidades odontológicas, Cassano et al. ${ }^{3}$, em 2002, realizaram um levantamento a partir de informaçóes do "site" de IBGE (Instituto Brasileiro de Geografia e Estatística) e junto aos Conselhos Federal e Estadual de Odontologia. Concluíram que o Paraná é um estado promissor para o mercado odontológico, pois $81 \%$ das cidades demonstraram uma relação $\mathrm{CD} /$ habitante menor que $1 / 1.500$. Afirmaram também que os municípios com maior relação $\mathrm{CD} /$ habitante eram Curitiba (444), Londrina (492) e Maringá (550), e que o mais atrativo foi São José dos Pinhais, com grande potencial econômico e próximo à capital.

O aumento do número de profissionais no mercado e a crescente queda da remuneração do cirurgião-dentista fizeram com que Sato ${ }^{21}$, em 2005, analisasse a situação da assistência odontológica suplementar, revisando a li- teratura atual, a legislação pertinente e os dados obtidos no setor. Foi concluído que há uma tendência para um rápido aumento do número desses profissionais nos próximos anos, não significando melhoria nas condiçóes de saúde bucal da população e no mercado de trabalho do $\mathrm{CD}$. Afirmou ainda que o faturamento do setor privado permanecerá estagnado, diferente do mercado odontológico suplementar, que aumenta a cada dia.

Junqueira et al. ${ }^{12}$ estudaram, ainda em 2005 , as alteraçôes no mercado de trabalho oriundas de mudanças na realidade social. O estudo utilizou como método a comparação de dados estatísticos do Conselho Federal de Odontologia (CFO), Instituto Brasileiro de Geografia e Estatística (IBGE) e Secretaria de Estado da Saúde de São Paulo. Foram verificadas diferenças na concentração de cirurgióes-dentistas por regióes, sendo que no sul e sudeste do país, onde se concentra a maior parte da renda do Brasil, está abrigado o maior número de universidades, além, também, do maior índice de cirurgiôesdentistas, demonstrando números distintos dos descritos pela Organização Mundial da Saúde (OMS). Os autores propóem como solução a interiorização, com o objetivo de reduzir essas discrepâncias regionais, assim como a busca por outros setores do mercado pouco explorados.

As recentes modificaçóes do mercado de trabalho do cirurgião-dentista nos estados brasileiros a partir de 1967, relacionadas à distribuição de profissionais clínicos gerais e ortodontistas, foram verificadas por Paranhos et al. ${ }^{16}$ (2008), visando, assim, melhorar a compreensão dos rumos da profissão. Concluíram que todas as capitais brasileiras apresentam índices maiores (menor número de habitantes por $\mathrm{CD}$ ) que o recomendado pela OMS, diferente do interior dos estados, onde se observou a proporção menor que 1:1.500. Para o especialista em Ortodontia, o interior dos estados também se mostrou mais promissor, principalmente nas regióes Norte e Nordeste.

\section{PROPOSIÇÃO}

O presente trabalho tem por objetivo avaliar, na Região Nordeste do Brasil, as modificaçóes que ocorreram na distribuição de cirurgióes-dentistas nas diferentes especialidades odontológicas, analisando a relação $\mathrm{CD} /$ habitante e especialista/habitante.

\section{MÉTODOS}

Os dados utilizados para a elaboração deste trabalho foram obtidos no "site" do Conselho Federal 
Paranhos LR, Ricci ID, Siqueira DF, Scanavini MA, Daruge Júnior E. Análise do mercado de trabalho odontológico na região nordeste do Brasil. Revista de Odontologia da Universidade Cidade de São Paulo 2009 mai-ago; 21(2): 104-18

de Odontologia 5 (2007), e do Instituto Brasileiro de Geografia e Estatística ${ }^{11}$, bem como dos relatórios emitidos pelo CFO. Através desses relatórios, obteveramse os números de cirurgióes-dentistas e de especialistas por Estado da Região Nordeste do Brasil, nos últimos 5 (cinco) anos.

No "site" do IBGE"11 foram colhidas informações relativas à população residente nos diferentes Estados. A partir dos dados coletados, foram calculadas as relaçóes especialista/habitante e CD/habitante, nos Estados da Regiâo Nordeste, comparados com o índice recomendado pela OMS, avaliando assim o mercado profissional odontológico.

\section{RESULTADOS E DISCUSSÃO}

A abertura de novos cursos de Odontologia teve grande crescimento perto dos anos 90, e, atualmente, encontram-se 188 cursos, concentrados em sua maior parte na Região Sudeste do Brasil (50,53\%), em especial no Estado de São Paulo (26,59\%), deixando a Região Nordeste com 15,95\% dos cursos, como mostra a Tabela 2.

A má distribuição do ensino no Brasil é um dos fatores responsáveis pela atual relação $\mathrm{CD}$ /habitante. $\mathrm{Na}$ Regiấo Nordeste, todos os Estados possuem maior proporção do que a sugerida pela OMS $(1: 1.500)$, com ex- ceção dos Estados da Paraíba e Rio Grande do Norte, que apresentam maior proporção entre habitantes por CD (Tabela 1).

Vários trabalhos mostram essa imparidade vivida pela Odontologia (Puricelli et al. ${ }^{19}$ 1999, Carvalho e Orlando ${ }^{2}$ 2001, Cassano et al. ${ }^{3}$ 2002, Garcia et al. ${ }^{9}$ 1997, Junqueira et al. ${ }^{12}$ 2005, Lara e Pereira Filho ${ }^{14} 1998$, Paranhos et al. ${ }^{16}$ 2008). Rocha et al. ${ }^{20}$ (1985). Em estudos qualitativos-descritivos do mercado de trabalho odontológico da Regiáo Nordeste do Brasil, esses autores comentaram que 728 municípios desta região não tinham assistência odontológica, deixando um campo aberto para o exercício ilegal da profissão, contrariando assim as leis do Brasil $^{1}$ (2003), em seu Art. 282. Junqueira et al. ${ }^{12}$ (2005) afirmaram que os profissionais, ao se formarem, buscam os grandes centros ou acabam se fixando próximos à região onde cursaram a graduação, talvez por facilidade ou pela busca cada vez maior de cursos de especialização.

De fato, a especialização e a constante capacitação profissional são fundamentais (Carvalho e Orlando ${ }^{2}$ 2001, Junqueira et al. ${ }^{12}$ 2005) e também um dever para o cirurgião-dentista, previsto no Código de Ética Odontológica ${ }^{4}$ Em 2003, Peres et al. ${ }^{18}$ afirmaram haver 287 cursos de especialização na Regiáo Sudeste e, em cinco anos, o número ter praticamente dobrado, chegando atualmente a 588 cursos, conforme dados do Conselho

Tabela 1 - Proporção de cirurgião-dentista, clínico geral e especialistas, por habitante, nos Estados da Região Nordeste do Brasil.

\begin{tabular}{ccccccc}
\hline \hline & $\begin{array}{c}\text { TOTAL } \\
\text { CDs }\end{array}$ & $\begin{array}{c}\text { CDs } \\
\text { ESPECIALISTAS }\end{array}$ & $\begin{array}{c}\text { \% GERAL } \\
\text { CDs } \\
\text { BRASIL }\end{array}$ & $\begin{array}{c}\text { POPULAÇÃO } \\
\text { (IBGE 2007) }\end{array}$ & $\begin{array}{c}\text { CD: } \\
\text { Habitante }\end{array}$ & $\begin{array}{c}\text { CDSPECIALISTA: } \\
\text { Habitante }\end{array}$ \\
\hline ALAGOAS & 1.930 & 151 & $0,87 \%$ & 3.037 .103 & $1: 1.573,63$ & $1: 20.113,26$ \\
BAHIA & 7.500 & 601 & $3,40 \%$ & 14.080 .654 & $1: 1.877,42$ & $1: 23.428,71$ \\
CEARÁ & 4.489 & 396 & $2,03 \%$ & 8.185 .286 & $1: 1.823,41$ & $1: 20.669,91$ \\
MARANHÁO & 2.062 & 171 & $0,93 \%$ & 6.118 .995 & $1: 2.967,50$ & $1: 35.783,60$ \\
PARAÍBA & 2.890 & 227 & $1,31 \%$ & 3.641 .395 & $1: 1.260,01$ & $1: 16.041,39$ \\
PERNAMBUCO & 5.646 & 346 & $2,56 \%$ & 8.485 .386 & $1: 1.502,90$ & $1: 24.524,24$ \\
PIAUÍ & 1.739 & 118 & $0,79 \%$ & 3.032 .421 & $1: 1.743,77$ & $1: 25.698,48$ \\
RIO GRANDE & 2.430 & 240 & $1,10 \%$ & 3.013 .740 & $1: 1.240,22$ & $1: 12.557,25$ \\
DO NORTE & 1.291 & 189 & $0,59 \%$ & 1.939 .426 & $1: 1.502,27$ & $1: 10.261,51$ \\
SERGIPE & & $* 2.439$ & $13,59 \%$ & 51.534 .406 & $1: 1.719,13$ & $1: 21.129,32$ \\
TOTAL REGIÃO & 29.977 & 57.728 & $100 \%$ & 183.987 .291 & $1: 834,02$ & $1: 3.187,14$ \\
NORDESTE & & & & & & \\
TOTAL BRASIL & 220.604 & & & & &
\end{tabular}

*(4,22\% dos Especialistas do País) 
Paranhos LR, Ricci ID, Siqueira DF, Scanavini MA, Daruge Júnior E. Análise do mercado de trabalho odontológico na região nordeste do Brasil. Revista de Odontologia da Universidade Cidade de São Paulo 2009 mai-ago; 21(2): 104-18

Tabela 2 - Número de faculdades de Odontologia e de cursos de especializaçáo reconhecidos e credenciados, nos Estados da Região Nordeste do Brasil.

\begin{tabular}{cccccc}
\hline \hline & FACULDADES & $\begin{array}{c}\text { CURSOS } \\
\text { RECONHECIDOS }\end{array}$ & $\begin{array}{c}\text { CURSOS } \\
\text { CREDENCIADOS }\end{array}$ & $\begin{array}{c}\text { TOTAL de cursos } \\
\text { de Especialização }\end{array}$ & $\%$ \\
\hline ALAGOAS & 2 & 1 & 2 & 3 & $0,36 \%$ \\
BAHIA & 6 & 10 & 1 & 11 & $1,33 \%$ \\
CEARÁ & 3 & 22 & 14 & 36 & $4,34 \%$ \\
MARANHÃO & 3 & 1 & 9 & 10 & $1,20 \%$ \\
PARAÍBA & 3 & 2 & 2 & 4 & $0,48 \%$ \\
PERNAMBUCO & 4 & 8 & 14 & 22 & $2,65 \%$ \\
PIAUÍ & 4 & 2 & 3 & 5 & $0,60 \%$ \\
RIO GRANDE & 2 & - & 3 & 3 & $0,36 \%$ \\
DO NORTE & 3 & 3 & - & 3 & $0,36 \%$ \\
SERGIPE & 30 & 49 & 48 & 97 & $11,69 \%$ \\
TOTAL REGIÁO & 188 & 433 & 397 & 830 & $100 \%$ \\
NORDESTE & & & & & \\
TOTAL BRASIL & & & & & \\
\hline \hline
\end{tabular}

Quadro 1 - Proporção de crescimento do número de especialistas no Estado de Alagoas.

\begin{tabular}{|c|c|c|c|c|c|c|}
\hline \multicolumn{7}{|c|}{ ALAGOAS - POPULAÇÃO 3.037 .103} \\
\hline \multirow{2}{*}{ ESPECIALIDADE ODONTOLÓGICA } & \multicolumn{5}{|c|}{ NÚMERO DE INSCRITOS ACUMULADOS } & \multirow{2}{*}{$\begin{array}{c}\text { PROPORÇÃO } \\
\text { ESPECIALISTA:HABITANTE }\end{array}$} \\
\hline & 2003 & 2004 & 2005 & 2006 & TOTAL 2007 & \\
\hline CIRURGIAE TRAUMATOLOGIA BUCO-MAXILO-FACIAIS & 1 & 4. & 6 & 6 & 8 & $1: 379637,88$ \\
\hline DENTÍSTICA & 7 & 8 & 10 & 12 & 12 & $1: 253091,92$ \\
\hline ENDODONTIA & 7 & 10 & 11 & 16 & 21 & $1: 144623,95$ \\
\hline 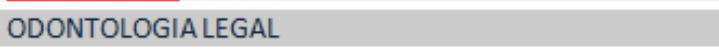 & - & - & 2 & 2 & 2 & $1: 1518551,50$ \\
\hline ODONTOPEDIATRIA & 4 & 7 & 7 & 13 & 15 & $1: 202473,53$ \\
\hline ORTODONTIA & 4 & 4 & 17 & 20 & 28 & $1: 108467,96$ \\
\hline PATOLOGIABUCAL & - & - & - & - & - & - \\
\hline PERIODONTIA & 3 & 3 & 9 & 9 & 9 & $1: 337455,89$ \\
\hline PRÓTESE BUCO-MAXILO-FACIAL & - & - & - & - & - & - \\
\hline PRÓTESE DENTÁRIA & - & 7 & 10 & 12 & 18 & $1: 168727,94$ \\
\hline IMPLANTODONTIA & 1 & 5 & 8 & 10 & 10 & $1: 303710,30$ \\
\hline ESTOMATOLOGIA & - & - & 1. & 1. & 4 & $1: 759275,75$ \\
\hline SAÚDE COLETIVA & - & - & - & 2 & 4 & $1: 759275,75$ \\
\hline RADIOLOGIAODONTÓLOGICAEIMAGINOLOGIA & - & - & 1 & 4 & 4 & $1: 759275,75$ \\
\hline DISFUNÇÃOTÊMPORO-MANDIBULAR E DOR-OROFACIAL & 1. & 4 & 5 & 5 & 5 & $1: 607420,60$ \\
\hline ODONTOLOGIADOTRABALHO & 1. & 1 & 1 & 1. & 1 & 1:3037103 \\
\hline ODONTOLOGIA PARA PACIENTESCOM NECES. ESPECIAIS & 1. & 2. & 2 & 2 & 2 & 1:1518551,50 \\
\hline ODONTOGERIATRIA & 1 & 1 & 1 & 1 & 1 & $1: 3037103$ \\
\hline ORTOPEDIA FUNCIONAL DOS MAXILARES & 6 & 7 & 7 & 7 & 7 & $1: 433871,86$ \\
\hline
\end{tabular}


Paranhos LR, Ricci ID, Siqueira DF, Scanavini MA, Daruge Júnior E. Análise do mercado de trabalho odontológico na região nordeste do Brasil. Revista de Odontologia da Universidade Cidade de São Paulo 2009 mai-ago; 21(2): 104-18

\begin{tabular}{|c|c|c|c|c|c|c|}
\hline \multicolumn{7}{|c|}{ BAHIA - POPULAÇÃO 14.080 .654} \\
\hline \multirow[t]{2}{*}{ ESPECIALIDADE ODONTOLÓGICA } & \multicolumn{5}{|c|}{ NÚMERO DE INSCRITOS ACUMULADOS } & \multirow{2}{*}{$\begin{array}{c}\text { PROPORÇÃO } \\
\text { ESPECIALISTA:HABITANTE }\end{array}$} \\
\hline & 2003 & 2004 & 2005 & 2006 & TOTAL 2007 & \\
\hline CIRURGIAE TRAUMATOLOGIA BUCO-MAXILO-FACIAIS & 8 & 13 & 16 & 23 & 30 & $1: 469355,13$ \\
\hline DENTÍSTICA & 6 & 11 & 19 & 26 & 30 & $1: 469355,13$ \\
\hline ENDODONTIA & 14 & 28 & 37 & 69 & 80 & $1: 176008,18$ \\
\hline ODONTOLOGIALEGAL & 2 & 4 & 4 & 4 & 5 & $1: 2816130,80$ \\
\hline ODONTOPEDIATRIA & 8 & 15 & 26 & 35 & 44 & $1: 320014,86$ \\
\hline ORTODONTIA & 13 & 25 & 55 & 92 & 114 & 1:123514,51 \\
\hline PATOLOGIABUCAL & 1 & 2 & 3 & 3 & 3 & $1: 4693551,33$ \\
\hline PERIODONTIA & 8 & 18 & 24 & 34 & 49 & $1: 287360,29$ \\
\hline PRÓTESE BUCO-MAXILO-FACIAL & - & - & - & - & - & - \\
\hline PROTESE DENTÁRIA & 12 & 27 & 47 & 64 & 86 & 1:163728,53 \\
\hline IMPLANTODONTIA & 1 & 1. & 3. & 10 & 18 & $1: 782258,56$ \\
\hline ESTOMATOLOGIA & 2 & 2 & 2 & 3 & 4 & $1: 3520163,50$ \\
\hline SAÚDE COLETIVA & 1 & 1 & 4 & 12 & 15 & $1: 938710,27$ \\
\hline RADIOLOGIAODONTÓLOGICAEIMAGINOLOGIA & 5 & 13 & 20 & 35 & 38 & $1: 370543,53$ \\
\hline DISFUNÇÃOTÊMPORO-MANDIBULAR E DOR-OROFACIAL & 7 & 9 & 9 & 9 & 12 & $1: 1173387,83$ \\
\hline ODONTOLOGIADOTRABALHO & - & - & - & - & - & - \\
\hline ODONTOLOGIA PARA PACIENTESCOM NECES. ESPECIAIS & 11 & 12 & 12 & 12 & 12 & 1:1173387,83 \\
\hline ODONTOGERIATRIA & 3 & 4 & 4. & 4 & 4 & $1: 3520163,50$ \\
\hline ORTOPEDIAFUNCIONAL DOS MAXILARES & 55 & 55 & 55 & 55 & 57 & $1: 247029,02$ \\
\hline
\end{tabular}

ESPECIALIDADE COM MAIOR NÜMERO DE INSCRITOS NO ESTADO ESPECIALIDADE COM MENOR NÚMERO DE INSCRITOS NO ESTADO

Quadro 2 - Proporção de crescimento do número de especialistas no Estado da Bahia.

Federal de Odontologia ${ }^{5}$ (2007), mostrando mais uma vez a má distribuiçáo do ensino.

Os cursos de Lato e Stricto Sensu também se multiplicaram vertiginosamente. No território brasileiro, distribuídos de forma desigual, existem 397 cursos de especialização credenciados (em andamento nas entidades de classe) e 433 cursos de especialização reconhecidos (em andamento nas faculdades), nas diferentes especialidades odontológicas (Conselho Federal de Odontologia ${ }^{5}$ 2007).

Assim, os nove Estados da Região Nordeste possuem 4,22\% dos especialistas do Brasil, nas diferentes áreas reconhecidas pelo Conselho Federal de Odontologia, como mostra a Tabela 2. A proporção especialista/habitante também é exposta nos Quadros 1 a 9, mostrando que a procura é maior por algumas especialidades, em particular a Ortodontia, a Endodontia e a Prótese Dentária, que lideram o "ranking", sendo bastante requisitadas nessa região. Em contrapartida, a Prótese Buco-Maxilo-Facial, a Odontologia Legal, a Patologia Bucal, a Odontologia do Trabalho e a Estomatologia são as especialidades menos procuradas, compreendendo campos de atuação que podem ser explorados. Eduardo ${ }^{6}$ (2008), em entrevista ao Jornal do CROSP, afirmou que o tratamento das manifestações bucais em pacientes submetidos à quimio e/ ou radioterapia está ganhando espaço no mercado odontológico, reafirmando, assim, que a Estomatologia é uma especialidade ainda promissora.

O Gráfico 1 mostra o número total de especialistas por gênero no Brasil, tendo como predominante o feminino (52\%) em quase todas as especialidades (Gráfico 2). Esse fato demonstra que as mulheres já são maioria no mercado odontológico, corroborando o estudo de Puricelli et al. ${ }^{19}$ (1999).

\section{CONCLUSÃO}

Baseados nos resultados obtidos, pode-se concluir que:

- Todos os Estados da Regiáo Nordeste do Brasil apresentam índices maiores que o recomendado pela Organização Mundial de Saúde (1:1.500), 
Paranhos LR, Ricci ID, Siqueira DF, Scanavini MA, Daruge Júnior E. Análise do mercado de trabalho odontológico na região nordeste do Brasil. Revista de Odontologia da Universidade Cidade de São Paulo 2009 mai-ago; 21(2): 104-18

\begin{tabular}{|c|c|c|c|c|c|c|}
\hline \multicolumn{7}{|c|}{ CEARÁ - POPULAÇĀO 8.185.286 } \\
\hline \multirow[t]{2}{*}{ ESPECIALIDADE ODONTOLÓGICA } & \multicolumn{5}{|c|}{ NÚMERO DE INSCRITOS ACUMULADOS } & \multirow{2}{*}{$\begin{array}{c}\text { PROPORÇÃO } \\
\text { ESPECIALISTA:HABITANTE }\end{array}$} \\
\hline & 2003 & 2004 & 2005 & 2006 & TOTAL 2007 & \\
\hline CIRURGIAETRAUMATOLOGIA BUCO-MAXILO-FACIAIS & - & 2. & 4 & 7 & 9 & $1: 909476,22$ \\
\hline DENTÍSTICA & 4 & 10 & 17 & 26 & 28 & $1: 292331,64$ \\
\hline ENDODONTIA & 19 & 31 & 46 & 62 & 69 & 1:118627,33 \\
\hline ODONTOLOGIALEGAL & - & - & - & - & - & - \\
\hline ODONTOPEDIATRIA & 4. & 9 & 19 & 36 & 44 & $1: 186029,22$ \\
\hline ORTODONTIA & 11 & 22 & 44 & 70 & 74 & $1: 110611,97$ \\
\hline PATOLOGIABUCAL & - & 1. & 1. & 2. & 2. & $1: 4092643$ \\
\hline PERIODONTIA & 13 & 21 & 28 & 37 & 41 & $1: 199641,12$ \\
\hline PRÓTESE BUCO-MAXILO-FACIAL & - & - & - & - & - & 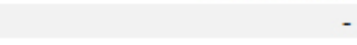 \\
\hline PRÓTESE DENTÁRIA & 5 & 16 & 27 & 47 & 54 & $1: 151579,37$ \\
\hline IMPLANTODONTIA & 3. & 5 & 10 & 13 & 17 & $1: 481487,41$ \\
\hline ESTOMATOLOGIA & - & 1. & 1 & 2 & 2 & $1: 4092643$ \\
\hline SAÚDE COLETIVA & - & - & 1. & 4. & 6 & $1: 1364214,30$ \\
\hline RADIOLOGIAODONTÓLOGICAEIMAGINOLOGIA & 2 & 5 & 6 & 9 & 9 & $1: 909476,22$ \\
\hline DISFUNÇÃOTÊMPORO-MANDIBULAR E DOR-OROFACIAL & 5 & 7 & 7 & 7 & 8 & $1: 1023160,70$ \\
\hline ODONTOLOGIADOTRABALHO & - & - & - & - & 1 & $1: 8185286$ \\
\hline ODONTOLOGIA PARA PACIENTESCOM NECES. ESPECIAIS & 2. & 2 & 2. & 2. & 3. & $1: 2728428,60$ \\
\hline ODONTOGERIATRIA & - & - & 1 & 1. & 2 & $1: 4092643$ \\
\hline ORTOPEDIA FUNCIONAL DOS MAXILARES & 20 & 24 & 25 & 26 & 27 & $1: 303158,74$ \\
\hline
\end{tabular}

$\square$ ESPECIALIDADE COM MAIOR NÚMERO DE INSCRITOS NO ESTADO

ESPECIALIDADE COM MENOR NÚMERO DE INSCRITOS NO ESTADO

Quadro 3 - Proporção de crescimento do número de especialistas no Estado do Ceará.

com exceção da Paraíba e do Rio Grande do Norte que apresentam maior proporção entre habitantes por CD;

- A Região Nordeste concentra $13,59 \%$ do total de cirurgióes-dentistas e 4,22\% dos especialistas de todo o Brasil;

- As especialidades com maior concorrência nessa região são Ortodontia, Endodontia e Prótese Dentária.

Assim, é necessário e importante que o cirurgiáo-dentista, antes de instalar seu consultório ou clínica odontológica, realize um levantamento da proporção de habitantes/profissionais para a escolha adequada do local, melhorando assim as chances de sucesso profissional. 
Paranhos LR, Ricci ID, Siqueira DF, Scanavini MA, Daruge Júnior E. Análise do mercado de trabalho odontológico na região nordeste do Brasil. Revista de Odontologia da Universidade Cidade de São Paulo 2009 mai-ago; 21(2): 104-18

\begin{tabular}{|c|c|c|c|c|c|c|}
\hline \multicolumn{7}{|c|}{ MARANHĀO - POPULAÇĀO 6.118.995 } \\
\hline \multirow[t]{2}{*}{ ESPECIALIDADE ODONTOLÓGICA } & \multicolumn{5}{|c|}{ NÚMERO DE INSCRITOS ACUMULADOS } & \multirow{2}{*}{$\begin{array}{c}\text { PROPORÇÃO } \\
\text { ESPECIALISTA:HABITANTE }\end{array}$} \\
\hline & 2003 & 2004 & 2005 & 2006 & TOTAL 2007 & \\
\hline CIRURGIAETRAUMATOLOGIA BUCO-MAXILO-FACIAIS & 2 & 2 & 2. & 3 & 4 & $1: 1529748,70$ \\
\hline DENTÍSTICA & 1 & 6 & 9 & 13 & 17 & $1: 359940,88$ \\
\hline ENDODONTIA & 5 & 12 & 16 & 16 & 16 & $1: 382437,18$ \\
\hline ODONTOLOGIALEGAL & - & - & - & - & - & 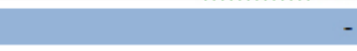 \\
\hline ODONTOPEDIATRIA & 12 & 15 & 18 & 21 & 27 & $1: 226629,44$ \\
\hline ORTODONTIA & 4 & 15 & 19 & 25 & 31 & 1:197386, 93 \\
\hline PATOLOGIABUCAL & - & - & - & - & - & 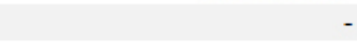 \\
\hline PERIODONTIA & - & 3 & 4 & 8 & 9 & 1:679888,33 \\
\hline PRÓTESE BUCO-MAXILO-FACIAL & - & - & - & - & - & \\
\hline PRÓTESE DENTÁRIA & 2 & 9 & 18 & 25 & 29 & 1:210999, 82 \\
\hline IMPLANTODONTIA & 2 & 7 & 9 & 9 & 10 & $1: 611899,50$ \\
\hline ESTOMATOLOGIA & - & - & - & - & - & \\
\hline SAÚDE COLETIVA & - & - & 1. & 3 & 4 & $1: 1529748,70$ \\
\hline RADIOLOGIAODONTÓLOGICAEIMAGINOLOGIA & 2. & 2 & 3 & 3 & 5 & $1: 1223799$ \\
\hline DISFUNÇÃOTÊMPORO-MANDIBULAR E DOR-OROFACIAL & 6 & 7 & 7 & 7 & 7 & $1: 874142,14$ \\
\hline ODONTOLOGIADOTRABALHO & 3. & 3 & 3 & 3 & 3 & $1: 2039665$ \\
\hline ODONTOLOGIA PARA PACIENTESCOM NECES. ESPECIAIS & 3 & 3 & 3 & 3 & 3 & $1: 2039665$ \\
\hline ODONTOGERIATRIA & - & - & - & - & - & \\
\hline ORTOPEDIA FUNCIONAL DOS MAXILARES & 6 & 6 & 6 & 6 & 6 & $1: 1019832,50$ \\
\hline
\end{tabular}

ESPECIALIDADE COM MAIOR NÜMERO DE INSCRITOS NO ESTADO

ESPECIALIDADE COM MENOR NÚMERO DE INSCRITOS NO ESTADO

Quadro 4- Proporção de crescimento do número de especialistas no Estado do Maranhão. 
Paranhos LR, Ricci ID, Siqueira DF, Scanavini MA, Daruge Júnior E. Análise do mercado de trabalho odontológico na região nordeste do Brasil. Revista de Odontologia da Universidade Cidade de São Paulo 2009 mai-ago; 21(2): 104-18

\begin{tabular}{|c|c|c|c|c|c|c|}
\hline \multicolumn{7}{|c|}{ PARAIIBA - POPULAÇĀO 3.641.395 } \\
\hline \multirow[t]{2}{*}{ ESPECIALIDADE ODONTOLÓGICA } & \multicolumn{5}{|c|}{ NÚMERO DE INSCRITOS ACUMULADOS } & \multirow{2}{*}{$\begin{array}{c}\text { PROPORÇÃO } \\
\text { ESPECIALISTA:HABITANTE }\end{array}$} \\
\hline & 2003 & 2004 & 2005 & 2006 & TOTAL 2007 & \\
\hline CIRURGIAETRAUMATOLOGIA BUCO-MAXILO-FACIAIS & - & 4 & 4 & 5 & 11 & $1: 331035,90$ \\
\hline DENTÍSTICA & 5 & 8 & 9 & 13 & 15 & 1:241759,66 \\
\hline ENDODONTIA & 7 & 8 & 11 & 20 & 26 & $1: 140053,65$ \\
\hline ODONTOLOGIALEGAL & - & - & - & - & - & - \\
\hline ODONTOPEDIATRIA & 2 & 11 & 13 & 16 & 20 & $1: 182069,75$ \\
\hline ORTODONTIA & 4 & 10 & 26 & 34 & 38 & $1: 95826,18$ \\
\hline PATOLOGIABUCAL & 2 & 3 & 3 & 4 & 4. & $1: 910348,75$ \\
\hline PERIODONTIA & 9 & 12 & 13 & 15 & 24 & $1: 151724,79$ \\
\hline PRÓTESE BUCO-MAXILO-FACIAL & - & - & - & - & - & - \\
\hline PRÓTESE DENTÁRIA & 15 & 16 & 17 & 24 & 28 & $1: 130049,82$ \\
\hline IMPLANTODONTIA & 2 & 2 & 5 & 10 & 13 & $1: 280107,30$ \\
\hline ESTOMATOLOGIA & 1 & 1 & 1 & 3 & 4 & $1: 910348,75$ \\
\hline SAÚDE COLETIVA & - & 2 & 7 & 10 & 14 & $1: 260099,64$ \\
\hline RADIOLOGIAODONTÓLOGICAE IMAGINOLOGIA & - & 3 & 4 & 6 & 10 & $1: 364139,50$ \\
\hline DISFUNÇÃOTÊMPORO-MANDIBULAR E DOR-OROFACIAL & 8 & 11 & 11 & 12 & 12 & $1: 303449,58$ \\
\hline ODONTOLOGIA DOTRABALHO & - & - & - & - & - & - \\
\hline ODONTOLOGIAPARA PACIENTESCOM NECES. ESPECIAIS & - & - & - & - & - & - \\
\hline ODONTOGERIATRIA & 1 & 2 & 2 & 2 & 2 & $1: 1820697,50$ \\
\hline ORTOPEDIA FUNCIONAL DOS MAXILARES & 4 & 4 & 4 & 5 & 6 & $1: 606899,16$ \\
\hline
\end{tabular}

Quadro 5 - Proporção de crescimento do número de especialistas no Estado da Paraíba. 
Paranhos LR, Ricci ID, Siqueira DF, Scanavini MA, Daruge Júnior E. Análise do mercado de trabalho odontológico na região nordeste do Brasil. Revista de Odontologia da Universidade Cidade de São Paulo 2009 mai-ago; 21(2): 104-18

\begin{tabular}{|c|c|c|c|c|c|c|}
\hline \multicolumn{7}{|c|}{ PERNAMBUCO - POPULAÇĀO 8.485.386 } \\
\hline \multirow{2}{*}{ ESPECIALIDADE ODONTOLÓGICA } & \multicolumn{5}{|c|}{ NÚMERO DE INSCRITOS ACUMULADOS } & \multirow{2}{*}{$\begin{array}{c}\text { PROPORÇÃO } \\
\text { ESPECIALISTA:HABITANTE }\end{array}$} \\
\hline & 2003 & 2004 & 2005 & 2006 & TOTAL 2007 & \\
\hline CIRURGIAETRAUMATOLOGIA BUCO-MAXILO-FACIAIS & 3. & 6 & 6 & 12 & 16 & $1: 530336,62$ \\
\hline DENTÍSTICA & 4 & 10 & 13 & 17 & 28 & $1: 303049,50$ \\
\hline ENDODONTIA & 14 & 24 & 31 & 39 & 46 & $1: 184469,41$ \\
\hline ODONTOLOGIALEGAL & 1 & 1 & 1. & 3 & 9 & $1: 942820,66$ \\
\hline ODONTOPEDIATRIA & 8 & 14 & 17 & 23 & 31 & $1: 273722,12$ \\
\hline ORTODONTIA & 7 & 18 & 48 & 57 & 71 & $1: 119512,47$ \\
\hline PATOLOGIABUCAL & 1. & 2 & 2 & 2 & 2 & $1: 4242693$ \\
\hline PERIODONTIA & 17 & 23 & 32 & 40 & 48 & $1: 176778,87$ \\
\hline PRÓTESE BUCO-MAXILO-FACIAL & - & - & - & - & - & - \\
\hline PRÓTESE DENTÁRIA & - & 2 & 13 & 20 & 26 & $1: 326361$ \\
\hline IMPLANTODONTIA & 5 & 5 & 12 & 17 & 22 & $1: 385699,36$ \\
\hline ESTOMATOLOGIA & 1. & 1. & 1. & 1. & 2. & $1: 4242693$ \\
\hline SAÚDE COLETIVA & 1. & 1. & 1. & 2 & 4. & $1: 2121346,50$ \\
\hline RADIOLOGIA ODONTÓLOGICAEIMAGINOLOGIA & 3 & 3 & 4 & 10 & 12 & $1: 707115,50$ \\
\hline DISFUNÇÃOTÊMPORO-MANDIBULAR E DOR-OROFACIAL & 5 & 5 & 5 & 6 & 7 & 1:1212198 \\
\hline ODONTOLOGIADOTRABALHO & - & - & - & - & - & - \\
\hline ODONTOLOGIA PARA PACIENTESCOM NECES. ESPECIAIS & 2. & 5 & 5 & 5 & 5 & $1: 1697077,20$ \\
\hline ODONTOGERIATRIA & 1 & 1 & 1 & 1 & 1 & $1: 8485386$ \\
\hline ORTOPEDIA FUNCIONAL DOS MAXILARES & 13 & 16 & 16 & 16 & 16 & $1: 530336,62$ \\
\hline
\end{tabular}

Quadro 6 - Proporção de crescimento do número de especialistas no Estado do Pernambuco. 
Paranhos LR, Ricci ID, Siqueira DF, Scanavini MA, Daruge Júnior E. Análise do mercado de trabalho odontológico na região nordeste do Brasil. Revista de Odontologia da Universidade Cidade de São Paulo 2009 mai-ago; 21(2): 104-18

\begin{tabular}{|c|c|c|c|c|c|c|}
\hline \multicolumn{7}{|c|}{ PIAUÍ - POPULAÇÃO 3.032.421 } \\
\hline \multirow{2}{*}{ ESPECIALIDADE ODONTOLÓGICA } & \multicolumn{5}{|c|}{ NÚMERO DE INSCRITOS ACUMULADOS } & \multirow{2}{*}{$\begin{array}{c}\text { PROPORÇÃO } \\
\text { ESPECIALISTA:HABITANTE }\end{array}$} \\
\hline & 2003 & 2004 & 2005 & 2006 & TOTAL 2007 & \\
\hline CIRURGIAETRAUMATOLOGIA BUCO-MAXILO-FACIAIS & 2. & 2. & 2. & 2. & 3 & $1: 1010807$ \\
\hline DENTÍSTICA & 5 & 8 & 9 & 12 & 13 & $1: 233263,15$ \\
\hline ENDODONTIA & 10 & 13 & 16 & 22 & 24 & $1: 126350,87$ \\
\hline ODONTOLOGIALEGAL & - & - & - & - & - & - \\
\hline ODONTOPEDIATRIA & - & 2. & 2 & 2. & 3 & 1:1010807 \\
\hline ORTODONTIA & 2 & 11 & 16 & 26 & 28 & $1: 108300,75$ \\
\hline PATOLOGIABUCAL & - & - & - & - & - & \\
\hline PERIODONTIA & 8 & 13 & 15 & 18 & 20 & $1: 151621,05$ \\
\hline PRÓTESE BUCO-MAXILO-FACIAL & - & - & - & - & - & \\
\hline PRÓTESE DENTÁRIA & 2 & 4 & 6 & 9 & 13 & $1: 233263,15$ \\
\hline IMPLANTODONTIA & - & - & 1. & 3 & 6 & $1: 505403,50$ \\
\hline ESTOMATOLOGIA & - & - & - & - & - & - \\
\hline SAÚDE COLETIVA & - & - & - & - & 2. & $1: 1516210,50$ \\
\hline RADIOLOGIAODONTÓLOGICAEIMAGINOLOGIA & 1 & 1. & 1. & 2. & 2. & $1: 1516210,50$ \\
\hline DISFUNÇÃOTÊMPORO-MANDIBULAR E DOR-OROFACIAL & 1. & 1. & 1. & 1. & 1. & $1: 3032421$ \\
\hline ODONTOLOGIADOTRABALHO & - & - & - & - & - & - \\
\hline ODONTOLOGIA PARA PACIENTESCOM NECES. ESPECIAIS & - & - & - & - & - & - \\
\hline ODONTOGERIATRIA & 1 & 1 & 1. & 1. & 1. & $1: 3032421$ \\
\hline ORTOPEDIAFUNCIONAL DOS MAXILARES & - & - & - & 2 & 2 & $1: 1516210,50$ \\
\hline
\end{tabular}

Quadro 7 - Proporção de crescimento do número de especialistas no Estado do Piauí. 
Paranhos LR, Ricci ID, Siqueira DF, Scanavini MA, Daruge Júnior E. Análise do mercado de trabalho odontológico na região nordeste do Brasil. Revista de Odontologia da Universidade Cidade de São Paulo 2009 mai-ago; 21(2): 104-18

\begin{tabular}{|c|c|c|c|c|c|c|}
\hline \multicolumn{7}{|c|}{ RIO GRANDE DO NORTE - POPULAÇAO 3.013.370 } \\
\hline \multirow[t]{2}{*}{ ESPECIALIDADE ODONTOLÓGICA } & \multicolumn{5}{|c|}{ NÚMERO DE INSCRITOS ACUMULADOS } & \multirow{2}{*}{$\begin{array}{c}\text { PROPORÇÃO } \\
\text { ESPECIALISTA:HABITANTE }\end{array}$} \\
\hline & 2003 & 2004 & 2005 & 2006 & TOTAL 2007 & \\
\hline CIRURGIAE TRAUMATOLOGIABUCO-MAXILO-FACIAIS & - & 1. & 3 & 4 & 8 & $1: 376671,25$ \\
\hline DENTÍSTICA & 3 & 9 & 11 & 13 & 15 & $1: 200891,33$ \\
\hline ENDODONTIA & - & 12 & 17 & 24 & 32 & $1: 94167,81$ \\
\hline ODONTOLOGIALEGAL & - & - & - & - & - & - \\
\hline ODONTOPEDIATRIA & 2 & 4 & 6 & 12 & 15 & $1: 200891,33$ \\
\hline ORTODONTIA & 10 & 13 & 26 & 42 & 55 & $1: 54788,54$ \\
\hline PATOLOGIABUCAL & - & 2 & 2 & 2 & 2 & $1: 1506685$ \\
\hline PERIODONTIA & 5 & 13 & 17 & 26 & 30 & 1:100445,66 \\
\hline PRÓTESE BUCO-MAXILO-FACIAL & - & - & - & 2 & 2 & $1: 1506685$ \\
\hline PRÓTESE DENTÁRIA & 5 & 15 & 24 & 33 & 36 & $1: 83704,72$ \\
\hline IMPLANTODONTIA & 2. & 4 & 4 & 6 & 16 & 1:188335,62 \\
\hline ESTOMATOLOGIA & - & - & - & - & - & - \\
\hline SAÚDE COLETIVA & - & - & 2 & 5 & 8 & $1: 376671,25$ \\
\hline RADIOLOGIA ODONTÓLOGICAE IMAGINOLOGIA & - & 1. & 2 & 5 & 7 & $1: 430481,42$ \\
\hline DISFUNÇÃOTÊMPORO-MANDIBULAR E DOR-OROFACIAL & 3. & 3. & 3. & 3 & 3 & $1: 1004456,60$ \\
\hline ODONTOLOGIADOTRABALHO & - & - & - & - & - & - \\
\hline ODONTOLOGIA PARA PACIENTES COM NECES. ESPECIAIS & 1 & 1. & 1. & 1. & 1. & $1: 3013370$ \\
\hline ODONTOGERIATRIA & - & - & - & - & - & - \\
\hline ORTOPEDIA FUNCIONAL DOS MAXILARES & 4 & 8 & 9 & 9 & 9 & $1: 334818,88$ \\
\hline
\end{tabular}

Quadro 8 - Proporção de crescimento do número de especialistas no Estado do Rio Grande do Norte. 
Paranhos LR, Ricci ID, Siqueira DF, Scanavini MA, Daruge Júnior E. Análise do mercado de trabalho odontológico na região nordeste do Brasil. Revista de Odontologia da Universidade Cidade de São Paulo 2009 mai-ago; 21(2): 104-18

\begin{tabular}{|c|c|c|c|c|c|c|}
\hline \multicolumn{7}{|c|}{ SERGIPE - POPULAÇÃO 1.939.426 } \\
\hline \multirow[t]{2}{*}{ ESPECIALIDADE ODONTOLÓGICA } & \multicolumn{5}{|c|}{ NÚMERO DE INSCRITOS ACUMULADOS } & \multirow{2}{*}{$\begin{array}{c}\text { PROPORÇÃO } \\
\text { ESPECIALISTA:HABITANTE }\end{array}$} \\
\hline & 2003 & 2004 & 2005 & 2006 & TOTAL 2007 & \\
\hline \multirow[t]{2}{*}{ CIRURGIAE TRAUMATOLOGIA BUCO-MAXILO-FACIAIS } & & & & & & $1: 242428,25$ \\
\hline & - & 1. & 3. & 4. & 8 & \\
\hline DENTÍSTICA & 1. & 7 & 10 & 23 & 26 & $1: 74593,31$ \\
\hline ENDODONTIA & 3. & 4 & 4 & 14 & 16 & $1: 121214,12$ \\
\hline ODONTOLOGIALEGAL & - & - & - & - & - & - \\
\hline ODONTOPEDIATRIA & 2. & 4. & 6 & 6 & 6 & $1: 323237,66$ \\
\hline ORTODONTIA & 4 & 8 & 17 & 29 & 33 & $1: 58770,48$ \\
\hline PATOLOGIABUCAL & 2. & 2 & 2 & 2 & 2 & 1:969713 \\
\hline PERIODONTIA & 3 & 4 & 4 & 8 & 10 & $1: 193942,60$ \\
\hline PRÓTESE BUCO-MAXILO-FACIAL & - & - & - & - & - & - \\
\hline PRÓTESE DENTÁRIA & 10 & 10 & 13 & 20 & 20 & $1: 96971,30$ \\
\hline IMPLANTODONTIA & - & - & - & - & 2 & $1: 969713$ \\
\hline ESTOMATOLOGIA & - & 1 & 1 & 1 & 2 & $1: 969713$ \\
\hline SAUDDE COLETIVA & 1 & 2 & 5 & 17 & 27 & $1: 71830,59$ \\
\hline RADIOLOGIAODONTÓLOGICAEIMAGINOLOGIA & 1 & 5 & 6 & 7 & 8 & $1: 242428,25$ \\
\hline $\begin{array}{l}\text { DISFUNÇÃOTÊMPORO-MANDIBULAR E DOR- } \\
\text { OROFACIAL }\end{array}$ & 3 & 4 & 4 & 4 & 4 & $1: 484856,50$ \\
\hline ODONTOLOGIADOTRABALHO & 3 & 3 & 3 & 4 & 4 & $1: 484856,50$ \\
\hline $\begin{array}{l}\text { ODONTOLOGIA PARA PACIENTESCOM NECES. } \\
\text { ESPECIAIS }\end{array}$ & 3 & 3 & 3 & 3 & 3 & $1: 646475,33$ \\
\hline ODONTOGERIATRIA & 1 & 1 & 1 & 1 & 1. & $1: 1939426$ \\
\hline ORTOPEDIA FUNCIONAL DOS MAXILARES & 15 & 16 & 17 & 17 & 17 & $1: 114083,88$ \\
\hline
\end{tabular}

ESPECIALIDADE COM MAIOR NÚMERO DE INSCRITOS NO ESTADO

ESPECIALIDADE COM MENOR NÚMERO DE INSCRITOS NO ESTADO

Quadro 9 - Proporção de crescimento do número de especialistas no Estado do Sergipe.

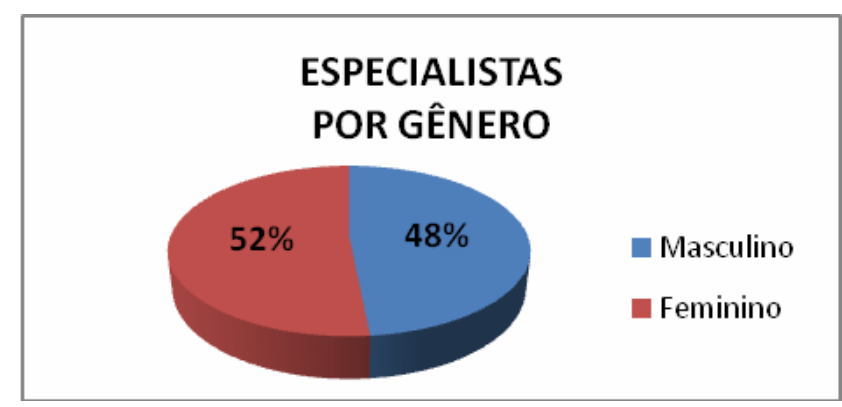

Gráfico 1 - Proporção de especialistas por gênero no Brasil. 
Paranhos LR, Ricci ID, Siqueira DF, Scanavini MA, Daruge Júnior E. Análise do mercado de trabalho odontológico na região nordeste do Brasil. Revista de Odontologia da Universidade Cidade de São Paulo 2009 mai-ago; 21(2): 104-18

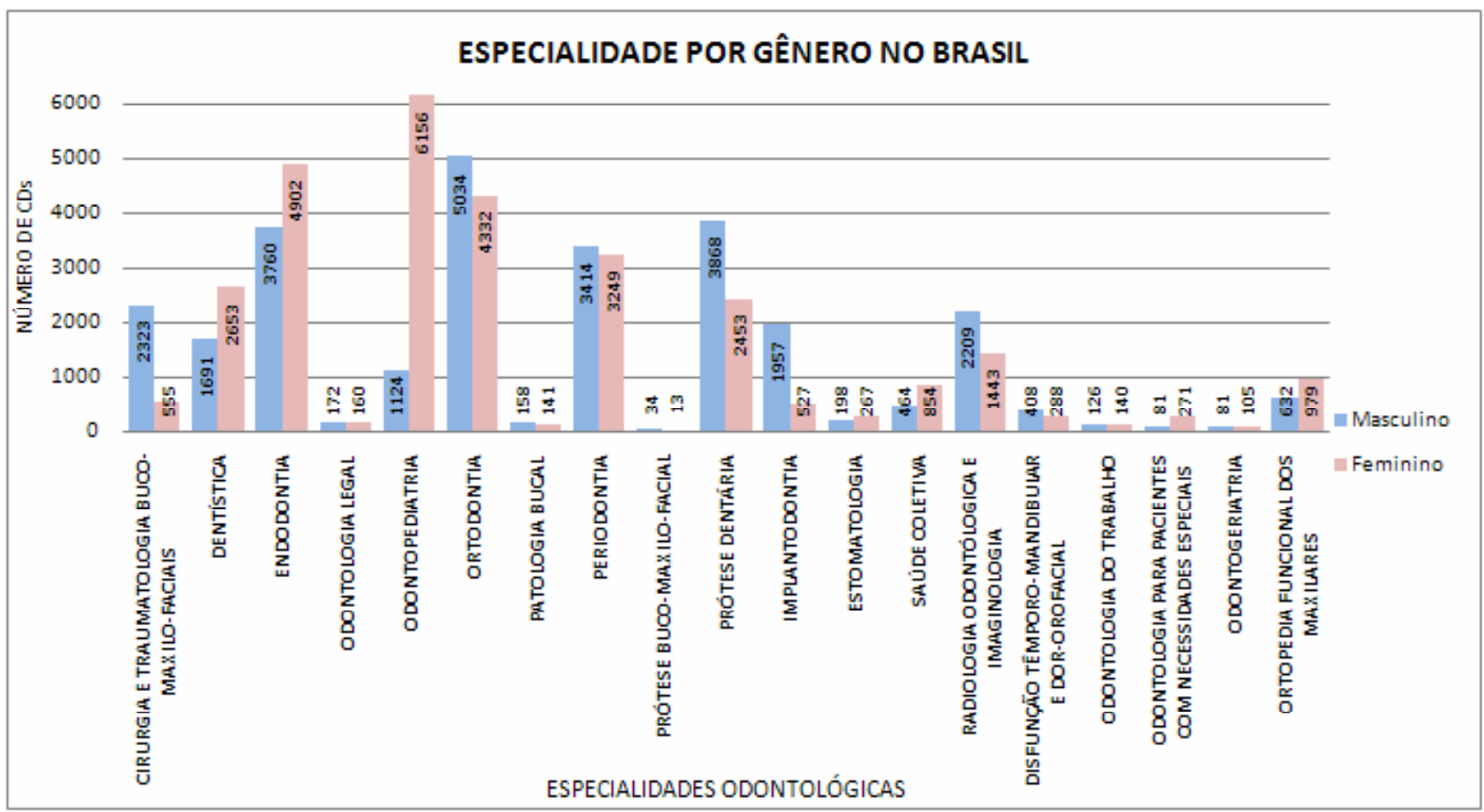

Gráfico 2 - Proporção de cirurgióes-dentistas, em cada especialidade, por gênero, no Brasil.

\section{REFERÊNCIAS}

1. Brasil. Leis e Decretos. Código penal: decreto lei ${ }^{\circ}$ 2.848, de 7 de dezembro de 1940. 8. ed. São Paulo: Saraiva; 2003.

2. Carvalho C, Orlando S. Futuro incerto. Rev Bras Odontol 2001 jan-fev; 58(1): 36-39.

3. Cassano DS, Telles CCC, Bonan RF, Freitas EM, Garcia PPNS, et al. Mercado de trabalho: avaliação da relação habitante/cirurgião-dentista no Estado do Paraná. Rev Odontol UNESP 2002 jan-jul; 31(1): 117-126.

4. Código de ética odontológica, Rio de Janeiro: Conselho Federal de Odontologia, 2006. Disponível em: <http://www.cfo.org.br/download/pdf/codigo_ etica.pdf>. Acesso em 02 de Junho 2008.

5. Conselho Federal de Odontologia (Brasil). Dados do CFO [homepage na Internet]. Disponível em: $<$ http://www.cfo.org.br>. Acesso em 02 de Junho 2008.

6. Eduardo CP. O mercado de trabalho do cirurgiãodentista só será ampliado facilitando o acesso da população à odontologia e explorando áreas com potencial de crescimento. Novo Crosp 2008 jan-mar; 27(220): 18-19.

7. Farias RL. Vão criar mais cursos? O estabelecimento de condutas éticas quando pelo estatuto de ABOR visa, entre outras coisas, o combate a proliferação desenfreada de cursos. Gazeta-ABOR 2006;7(2):1213.

8. Galassi MS, Santos-Pinto L, Scanavino FLF. Expectativas do cirurgião-dentista em relação ao mercado de trabalho. Rev Assoc Paul Cir Dent 2004 jan-fev; 58(1): 67-70.

9. Garcia PPNS, Corona SAM, Rosell FL, Porto FA, Castro JRF. Características do mercado de trabalho das principais cidades de Santa Catarina, de acordo com a proporção habitante/cirurgião-dentista. Odonto 2000 1997;1(2):28-31.

10. Gushi LL, Wada RS, Sousa MLR. Perfil profissional dos cirurgiões-dentistas formados pela FOB no período de 1960-1997. Rev Assoc Paul Cir Dent 2004 
Paranhos LR, Ricci ID, Siqueira DF, Scanavini MA, Daruge Júnior E. Análise do mercado de trabalho odontológico na região nordeste do Brasil. Revista de Odontologia da Universidade Cidade de São Paulo 2009 mai-ago; 21(2): 104-18

jan-fev; 58(1): 19-23.

11. Instituto Brasileiro de Geografia e Estatística IBGE [homepage na internet] (Brasil). Banco de Dados, Disponível em: < http://www.ibge.org.br>. Acesso em 02 de Junho 2008.

12. Junqueira CR, Ramos DLP, Rode SM. Considerações sobre o mercado de trabalho em odontologia. Rev Paul Odontol 2005 out-dez; 26(4): 24-27.

13. Koide RE, Paranhos LR, Quintela RS. Análise do perfil profissional na Odontologia. Rev Paul Odontol 2004 mai-jun; 26(3): 17-22.

14. Lara JCAG, Pereira Filho JB. Mercado de trabalho em Porto Alegre: distribuição geográfica dos consultórios dentários. Rev Odonto Ciênc 1998 dez; 13(26): 177-188.

15. Machado MH, Médici AC, Nogueira RP, Girardi SN. O mercado de trabalho em saúde no Brasil: estrutura e conjuntura. Rio de Janeiro: Ensp, 1992 p. 9-26.

16. Paranhos LR, Salazar M, Koide RE, Ramos AL. Análise do mercado de trabalho de cirurgiões-dentistas, clínicos gerais e especialistas em ortodontia, nos estados brasileiros. Rev Clin Ortodon Dental Press 2008 abr-mai; 7(2): 79-85.

17. Pereira MF, Botelho TL. Perfil do cirurgião-dentista no estado de Goiás parte I. Rev Fac Odontol UFG 1997;1(1):37-40.
18. Peres AS, Matos PES, Leal RB. Proliferação das especialidades odontológicas: uma abordagem crítica. Rev Odontol UNICID 2003 mai-ago; 15(2): 115-121.

19. Puricelli E, Eduardo MAP, Yurgel LS, Martinelli PM. A revolução silenciosa. Rev ABO Nac. 1999 ago-set; 7(4): 198-20.

20. Rocha MP, Costa DB, Sintes JL, Albuquerque AJ. Mercado de trabalho em odontologia no Nordeste do Brasil. RGO (Porto Alegre) 1985 out-dez; 33(4): 286-291.

21. Sato FRL. Mercado de assistência odontológica suplementar: situação atual e perspectivas futuras. Rev Assoc Paul Cir Dent 2005 jan-fev; 59(1): 37-41.

22. Silva Filho FPM, Eleutério D. Análise da remuneração paga aos dentistas em empregos públicos e privados. Rev Ass Paul Cirurg Dent 1977; 31(2): 69-72.

23. Sória ML, Bordin R, Costa Filho LC. Remuneração dos serviços de saúde bucal: formas e impactos na assistência. Cad Saúde Pública 2002 nov-dez; 18(6): 1551-1559.

Recebido em: 27/10/2008

Aceito em: 24/03/2009 